ACCEPTED MANUSCRIPT • OPEN ACCESS

\title{
Fe K-edge X-ray Absorption Spectroscopy of corrosion phases of archaeological iron: results, limitations, and the need for complementary techniques
}

To cite this article before publication: Hayley Simon et al $2021 \mathrm{~J}$. Phys.: Condens. Matter in press https://doi.org/10.1088/1361-648X/ac08b6

\section{Manuscript version: Accepted Manuscript}

Accepted Manuscript is "the version of the article accepted for publication including all changes made as a result of the peer review process, and which may also include the addition to the article by IOP Publishing of a header, an article ID, a cover sheet and/or an 'Accepted Manuscript' watermark, but excluding any other editing, typesetting or other changes made by IOP Publishing and/or its licensors"

This Accepted Manuscript is @ 2021 The Author(s). Published by IOP Publishing Ltd.

As the Version of Record of this article is going to be / has been published on a gold open access basis under a CC BY 3.0 licence, this Accepted Manuscript is available for reuse under a CC BY 3.0 licence immediately.

Everyone is permitted to use all or part of the original content in this article, provided that they adhere to all the terms of the licence https://creativecommons.org/licences/by/3.0

Although reasonable endeavours have been taken to obtain all necessary permissions from third parties to include their copyrighted content within this article, their full citation and copyright line may not be present in this Accepted Manuscript version. Before using any content from this article, please refer to the Version of Record on IOPscience once published for full citation and copyright details, as permissions may be required. All third party content is fully copyright protected and is not published on a gold open access basis under a CC BY licence, unless that is specifically stated in the figure caption in the Version of Record.

View the article online for updates and enhancements. 


\title{
Fe $K$-edge X-ray Absorption Spectroscopy of corrosion phases of archaeological iron: results, limitations, and the need for complementary
} techniques

\author{
Hayley Simon ${ }^{1,2}$, Giannantonio Cibin ${ }^{2}$, Ian Freestone ${ }^{1}$ and Eleanor Schofield ${ }^{3 *}$ \\ ${ }^{1}$ Institute of Archaeology, University College London, 31-34 Gordon Square, London, WC1H 0PY, UK \\ ${ }^{2}$ Diamond Light Source, Harwell Campus, Didcot, Oxfordshire, OX11 0DE, UK \\ ${ }^{3}$ The Mary Rose Trust, College Road, HM Naval Base, Portsmouth, PO1 3LX, UK \\ E-mail: E.Schofield@maryrose.org
}

Received xxxxxx

Accepted for publication $\mathrm{xxxxxx}$

Published xxxxxx

\begin{abstract}
Data analysis methods for iron X-ray Absorption Spectroscopy (XAS) can provide extensive information about the oxidation state and co-ordination of an Fe-species. However, the extent to which techniques developed using a single-phase iron sample may be applied to complex, mixed-phase samples formed under real-world conditions is not clear. This work uses a combination of pre-edge fitting and linear combination analysis (LCA) to characterise the near edge region of the X-ray absorption spectrum (XANES) for a set of archaeological iron corrosion samples from a collection of cast iron cannon shot excavated from the Mary Rose shipwreck and compares the data with phase compositions determined by Synchrotron X-ray Powder Diffraction (SXPD). Archaeological powder and cross-section samples were compared to a library of iron standards and diffraction data. The XANES are consistent with previous observations that generation of the chlorinated phase akaganeite, $\beta-\mathrm{FeO}(\mathrm{OH}, \mathrm{Cl})$, occurs in those samples which have been removed form passive storage and subjected to active conservation. However, the results show that if any metallic species is present in the sample, the contribution from $\mathrm{Fe}(0)$ to the spectral region containing a pre-edge for oxidised iron - Fe(II) and $\mathrm{Fe}(\mathrm{III})$ - causes the analysis to be less effective and the conclusions unreliable. Consequently, while the pre-edge fitting methodology may be applied to a mixture of iron oxides or oxyhydroxides, the procedure is inappropriate for a mixed metal-oxide sample without the application of a complimentary technique, such as SXPD.
\end{abstract}

Keywords: XANES, SXPD, iron, archaeology, corrosion, conservation, Mary Rose

\section{Introduction}

Corrosion occurs when a material degrades by reacting with an external environment. Over time, this process can cause significant damage to the corroding material, which may have devastating consequences if the material acts as a structural component or if the corroding object is a unique archaeological artefact. To prevent material loss, interventive treatments are required. However, the development of a corrosion inhibitor or conservation treatment relies on a 
fundamental understanding about how corrosion proceeds in a real-world environment.

Typically, corrosion progresses through a series of redox reactions that lead to oxidation of the corroding species. For metallic iron in a natural environment, this results in a substrate of $\mathrm{Fe}^{0}$ with overlying corrosion layers consisting of $\mathrm{Fe}^{2+}, \mathrm{Fe}^{3+}$ and mixed $\mathrm{Fe}^{2+/ 3+}$ compounds [1]. Characterisation of these layers can provide insights into how the reaction proceeds over time [2-4]. One methodology that may be used to determine the oxidation state of an element in a material is X-ray Absorption Spectroscopy (XAS); a powerful, elementspecific technique that can be applied to both crystalline and amorphous materials, in an isolated state or under reaction conditions. In the case of an iron species, examination of the Fe $K$-edge XAS is a highly effective tool for investigating iron oxidation states in minerals [5,6] and glasses [7,8] and has a long history of application in corrosion science [9-15]. In particular, analysis of the near edge region of the X-ray absorption spectrum (XANES) can provide a quantitative assessment of the ratio of $\mathrm{Fe}^{2+}$ to $\mathrm{Fe}^{3+}$ in a powder sample, through peak-fitting of the pre-edge feature of the XANES spectrum [5-8,16,17]. Previous studies have, however, focused on samples containing a single iron compound, either a laboratory-synthesised sample $[16,18]$, or a naturally formed mineral mixed with non-iron bearing species $[6,17]$. A greater challenge arises when applying $\mathrm{Fe} K$-edge XAS to complex samples containing multiple iron-species, such as a mixture of iron oxides formed during corrosion in a natural environment.

This paper investigates the extent to which iron XANES data analysis methodologies may be applied to complex, heterogeneous, mixed-phase samples that have been exposed to real-world environments that change over time. To achieve this, this work will examine a set of corroded archaeological iron samples from the Mary Rose shipwreck, previously studied [19] by Synchrotron X-ray Powder Diffraction (SXPD) and $\mathrm{Cl} K$-edge XAS. This sample set offered a unique opportunity to compare the corrosion products on iron artefacts with virtually identical burial histories, but which were subjected to different conservation regimes over the past four decades. The need for such a study arose from observations that, despite the use of widely applied conservation treatments, the Mary Rose artefacts continued to deteriorate, in some cases catastrophically. The investigation of these samples showed that the corrosion phases present on marine archaeological iron differ depending on the conservation history of the object, with long-term aqueous storage at $\mathrm{pH} 10$ completely removing $\mathrm{Cl}^{-}$ions from the surface of the artefact. However, sub-surface chloride remains trapped in metal pores and casting voids. After being removed from passive storage, trapped $\mathrm{Cl}^{-}$reacts during active conservation treatment, for example alkaline washing of the artefact, to form the chlorinated iron oxyhydroxide akaganeite, $\beta-\mathrm{FeO}(\mathrm{OH}, \mathrm{Cl})$, leading to degradation of the object [19,20]. Building on the earlier analysis, complementary X-ray absorption spectra from the Fe $K$-edge were collected from the same sample-set and used here to investigate the complex, mixed-species archaeological iron corrosion in comparison to a reference library of standard iron corrosion products and SXPD. The purpose of this work is to critically examine different data analysis methods for mixedphase Fe XAS spectra and to compare the results to the previously published data sets. From this, an exploration of the extent to which the diffraction and absorption spectra can be used independently and the advantages of using them in consort will be achieved, with a view to maximising the information that may be extracted from heterogenous samples formed in real-world environments.

\section{Methodology}

\subsection{Preparation of samples and standards}

Archaeological samples were collected from 19 cast iron cannon shot excavated between 1979 and 1983 from the Mary Rose (c. 1545) shipwreck site near Portsmouth, UK [21-23]. Immediately after excavation, all shot were immersed in high $\mathrm{pH}$ solution - either $\mathrm{NH}_{3}, \mathrm{NaOH}$, or an equimolar mixture of $\mathrm{Na}_{2} \mathrm{CO}_{3} / \mathrm{NaHCO}_{3}$ - but have since been treated by different methodologies. Consequently, the sampled shot were divided into 3 categories based on their different conservation history:

1) SS: passive storage in $0.15 \mathrm{M}(\mathrm{pH} 10)$ sodium sesquicarbonate $\left(\mathrm{Na}_{2} \mathrm{CO}_{3} / \mathrm{NaHCO}_{3}\right)$ solution until present, 11 shot

2) HW: same as (1) until 2010-12, when rinsed in tap water, washed in 3-4 consecutive baths of 1 or $2 \%$ $v: v$ corrosion inhibitor Hostacor® IT:water [24], $(\mathrm{pH} 4.5-8.5)$ and dried in 2-stage acetone:water (1:1 and 1:0) series. Now stored in a controlled environment (approximately $20{ }^{\circ} \mathrm{C}, 20 \% \mathrm{RH}$ ), 4 shot.

3) HWAS: same as (2) but underwent additional alkaline sulfide, $\mathrm{NaOH} / \mathrm{Na}_{2} \mathrm{SO}_{4}, \quad(\mathrm{pH}$ 12-13) reduction treatment [25]. Now stored in controlled (approximately $20^{\circ} \mathrm{C}, 20 \% \mathrm{RH}$ ) environment , 4 shot.

To study the corrosion process, 3 types of samples were collected from the shot: bulk corrosion powders from the object surface (-C samples), cut cross-sections mounted in polyester resin (-S samples), and powders collected during cutting (-P samples). A detailed description of the sampling procedure is available in [19]. A breakdown of all samples, based on both sample type and treatment history is as follows: SS = 21 -C samples, 2 -S samples; HW = 2 -C samples, 3 -P samples, 2 -S samples; HWAS = 3 -C samples, 2 -P samples, 2 -S samples.

A library of iron corrosion standards was prepared through a combination of laboratory synthesis and purchases from 
chemical vendors, Table 1. The standard phases were chosen based on commonly observed iron corrosion phases, namely the iron oxides and oxyhydroxides, plus additional stable $\mathrm{Fe}(\mathrm{II})$ and $\mathrm{Fe}(\mathrm{III})$ species with a range of anions, $\mathrm{Cl}^{-}, \mathrm{S}^{-}, \mathrm{NO}^{3-}$ , $\mathrm{PO}_{4}{ }^{3-}, \mathrm{SO}_{4}{ }^{2-}, \mathrm{CO}_{3}{ }^{2-}$, to provide a reference for different features that may occur in the XANES spectra. For standards made in the laboratory, all aqueous solutions were prepared using distilled water, $\mathrm{dH}_{2} \mathrm{O}$, from an ELGA PureLab OptionQ system (High Wycombe, UK). Centrifugation was performed using a Thermo Scientific Heraeus Multifuge 3SRT. Full details of the synthesis methods and characterisation of laboratory-prepared samples are given in the supplementary information.

\subsection{Data collection}

Synchrotron experiments were carried out at Diamond Light Source (Oxfordshire, UK) using the core-EXAFS beamline, B18 [26,27]. Powder samples (-C, -P and Fe standards) were prepared as pressed pellets, containing $5 \mathrm{mg}$ Fe-compound and $100 \mathrm{mg}$ cellulose filler. Standards consisted of a mixture of purchased and laboratory-synthesised compounds, as detailed in Table 1. All powder pellet samples were wrapped in Kapton tape and measured at ambient pressure and temperature in transmission mode. The beamline optics for transmission measurements included $\mathrm{Pt}$ coated mirrors, a $\mathrm{Si}(111)$ monochromator for X-ray energy selection and $\mathrm{Pt}$ coated harmonic rejection mirrors. Pellets were mounted on a holder in the beamline. The intensity of the incident X-ray beam, $I_{0}$, was measured using the first ion chamber filled with $\mathrm{N}_{2}(15 \%$ abs) for the $-\mathbf{C}$ and $-\mathbf{P}$ samples and filled with $\operatorname{Ar}(15 \%$ abs) for the standards. A second and third ion chamber, each filled with Ar gas (150 mbar, 70\% abs) were used to measure the intensity of the pellets, $I_{t}$, and reference Fe foil, $I_{\text {ref }}$, respectively at the Fe $K$-edge in the energy range 6920-7920 eV in steps of $0.25 \mathrm{eV}$. The spot size of the beam was $\sim 100 \times 100 \mu \mathrm{m}$. Six repeat scans were collected of each $-\mathbf{C}$ and $-\mathbf{P}$ sample spectrum, and Three repeat scans taken of each iron standard spectrum. All archaeological powder samples (-C and -P samples) were further characterised by SXPD at Diamond Light Source beamline I11, using the methodology described in $[19,20]$.

Additional Fe $K$-edge spectra were collected from 6 crosssection samples (-S samples) at ambient pressure and temperature in total electron yield, TEY, mode. An electrical contact for TEY was made with $\mathrm{Cu}$ wire. TEY signal was collected in drain current mode with $200 \mathrm{~V}$ polarisation applied to an extraction ring located upstream of the sample. Spectra were collected in line scans along each cross-section going from outer corrosion layer to the internal region of the artefact at $1 \mathrm{~mm}$ intervals by in the energy range 6920$7920 \mathrm{eV}$ with steps of $0.25 \mathrm{eV}$. To determine the appropriate location for each line scan, each cross-section (-S sample) was mapped using the X-ray Fluorescence (XRF) elemental mapping with a 4 element Si drift detector at $7.1 \mathrm{keV}$. Full details of the mapping parameters are available in the supplementary information. The raw map data was exported as a series of HDF5 files and processed into 2D element maps using in-house mapping software developed at the B18 beamline [28], written in Python 3.6. The elemental maps presented in Figure 7 show the Fe distribution of the 6 analysed -S samples.

\subsection{Calibration and normalisation}

All XAS data were processed using Athena, part of the Demeter software package [29]. For both samples and standards, repeat spectra were merged and $\mathrm{E}_{0}$ chosen as the first peak in the first derivative.

Repeat measurements were merged to one spectrum and calibrated based on the reference Fe foil measured either during acquisition of the sample spectrum (transmission measurements) or just prior to data collection (TEY measurements). Spectra were calibrated by setting $\mathrm{E}_{0}$ for the Fe reference - defined as the largest peak in the first derivative - to $7112 \mathrm{eV}$ and applying the same energy shift to the experimental data. The $\mu(E)$ spectra [where $\mu(E)=\ln \left(\frac{I_{0}}{I_{t}}\right)$ ] were normalised/with a $3^{\text {rd }}$ order polynomial and the background subtracted. $\mathrm{E}_{0}$ for the experimental spectra was chosen as the largest peak in the first derivative. The same preedge and normalisation range were used for all spectra of the same conservation treatment. A linear combination analysis, LCA, was performed for each sample spectrum using the standards library described in Table $\mathbf{1 .}$

\subsection{Pre-edge analysis}

Following normalisation and background subtraction, the pre-edge feature of the Fe standards and powder (-C and -P) samples was extracted from the rising edge of the spectrum by performing a spline interpolation using data $\sim 6 \mathrm{eV}$ above and below the pre-edge, Figure 1. The interpolated spline was then subtracted from the spectrum, to give the extracted preedge Figure 1, which was deconvoluted into 3 peaks using a peak-fitting script written in Python 3.6. Each pre-edge was fit with 3 pseudo-vogit functions, defined as a linear combination of Gaussian and Lorentzian peak shapes. A 20\% Lorentzian fraction was found to give the best fit to the data. During the fitting, the centroid positions, widths and intensities of all 3 peaks were allowed to vary. Averaged data was calculated for each pre-edge, namely the integrated intensity [sum of the integrated intensities of each fitted peak] and the overall centroid position [intensity-weighted average of the peak positions], based on the methodology of Wilke et al. [5]. The background subtraction and peak fitting Python script is provided in the supplementary information. 


\section{Results and Discussion}

Marine archaeological iron corrosion typically leads to the formation of a mixture of iron oxyhydroxides, $\alpha$ - $\beta$ - or $\gamma$ $\mathrm{FeO}(\mathrm{OH})$; alongside a spinel of either magnetite, $\mathrm{Fe}_{3} \mathrm{O}_{4}$, maghemite, $\gamma-\mathrm{Fe}_{2} \mathrm{O}_{3}$, or both; and additional phases from the burial environment, such as $\mathrm{SiO}_{2}$ or $\mathrm{CaCO}_{3}$ which form a hard, external concretion layer [4,30-35]. Characterisation of the corrosion layout of iron artefacts following marine excavation $[4,36]$ shows that the phases form in layers, typically of $\mathrm{Fe}(\mathrm{II})$ at the metal-corrosion interface, with $\mathrm{Fe}$ (III) developed below the concretion. Chlorine may be incorporated into the corrosion layers, either in the form of the $\mathrm{Fe}$ (II) hydroxychloride, $\beta-\mathrm{Fe}_{2}(\mathrm{OH})_{3} \mathrm{Cl}$ [37], or as the $\mathrm{Fe}(\mathrm{III})$ phase akaganeite, $\beta-\mathrm{FeO}(\mathrm{OH}, \mathrm{Cl})[38]$.

The sample set investigated in this work looks at a total of 30 powder samples from the surface of the objects (-C samples) or collected during cutting of cross-sections (-P samples) from 19 cast iron cannon shot from the Mary Rose shipwreck. Previously published SXPD compositions from the powder samples [19] have been renormalised in this investigation to include only the iron-containing species identified in the diffraction composition. This was done to allow for a direct comparison to the XAS spectra, Figure 2, which is selective for the iron-bearing phases in the samples. The archaeological samples were investigated through comparison to a known library of standard iron corrosion species. Spectra from the standards will be made available to download from the online B18 data repository [39].

\subsection{Phase quantification of Fe-species in powder archaeological samples}

The 30 corrosion powder samples from the Mary Rose cannon shot are all complex mixed-species samples, presenting with a range of compositions and variable ratios of iron phases, Figure 3. From both SXPD and XAS, the iron corrosion phases goethite, $\alpha-\mathrm{FeO}(\mathrm{OH})$; akaganeite, $\beta$ $\mathrm{FeO}(\mathrm{OH}, \mathrm{Cl})$; lepidocrocite, $\gamma-\mathrm{FeO}(\mathrm{OH})$; magnetite, $\mathrm{Fe}_{3} \mathrm{O}_{4}$; maghemite, $\gamma-\mathrm{Fe}_{2} \mathrm{O}_{3}$; and siderite, $\mathrm{FeCO}_{3}$ were identified in the samples, along with two metal phases: ferrite, $\alpha-\mathrm{Fe}$ and cementite, $\mathrm{Fe}_{3} \mathrm{C}$. To determine the weight composition of the Fe-species from the archaeological samples, the XAS spectra in Figure 2 were compared to the spectral library of iron standards (supplementary information S.2) using a linear combination analysis, LCA. In the LCA, it was found that an equally good fit - see supplementary information S.3 for an assessment of the fit quality - was achieved for both ferrite and cementite, both of which represent metal species that originates from the underlying alloy of the shot. As a result, it was not possible to distinguish between the two using XAS and, therefore, in Figure 2-a they should be considered interchangeable - but the two metal phases are easily differentiated in the SXPD data. The reverse occurred for the iron oxide phases magnetite and maghemite, which give the same structure in SXPD, but may be distinguished by XAS. Consequently, the SXPD wt fraction compositions in Figure 2-b for 'magnetite' (yellow) are likely a mixture of magnetite and maghemite. From this, it is clear that the two methods are highly complementary, a conclusion which has been reached by other authors looking at similar systems [3,40-42].

From Figure 2, it can be seen that the EXAFS of the SS shot appear broadly similar and, even though there are fewer spectra for HW and HWAS shot, more variation is present between the individual samples within each group, as illustrated by the variance accounted for by the first component in a principle component analysis, PCA, of the 3 data sets, Table 2. However, this result could be due to the SS dataset only containing -C samples, while HW and HWAS both include -P samples; powders collected during shot sectioning. A poor LCA fit was achieved to the -P samples, as demonstrated by the R-factors given in the supplementary information S.4. The lower quality of the model fit for -P samples suggests the presence of an additional unknown species not accounted for in the standards library, which reduces the reliability of the compositions determined for the -P samples

To directly compare XAS and SXPD, the results of the LCA fit were plotted against the phase proportions of the ironcontaining species identified in the diffraction data, Figure 4. While there is a reasonable agreement between iron-phase quantification with XAS and SXPD, the diffraction composition tended to predict a greater proportion of goethite, while the LCA results suggests more spinel species (magnetite/maghemite) in the samples. For the 8 samples containing akaganeite, a phase of particular interest to archaeological iron corrosion research, a good quantification agreement was reached by the two techniques, confirming previous observations that akaganeite is absent from those samples which had remained in passive solution but present in the corrosion of artefacts which had been removed and subjected to active conservation treatments [19]. This result shows that analysis of mixed-species Fe XANES spectra can aid the identification of akaganeite in a sample, even at low concentrations.

Surprisingly, the XAS model was unable to identify lepidocrocite in any spectra, a phase which was observed in the diffraction data. This could be due to the similarity of iron oxyhydroxide absorption spectra (supplementary information S.2), which have spectral features that may be easily distinguished in a single-phase system but add contributions to a mixed-phase spectrum at similar locations. This means that an equally good LCA model fit may be achieved for each iron oxyhydroxide, or using a combination of iron oxyhydroxides, and the preferential selection of one phase over the other requires a complementary characterisation 
method, such as diffraction, to be used alongside the XAS analysis.

\subsection{Pre-edge analysis}

To further investigate the XANES data from -C and -P samples, a peak-fitting procedure was developed (available for use in supplementary information S.7) to study the preedge feature observed in the spectra. Analysis of the pre-edge feature in iron XANES can provide information on the coordination and oxidation state of an Fe mineral, for both crystalline [5,6] and amorphous [7,8,18,43] species. Plotting the integrated pre-edge intensity vs. the centroid position, it is possible to estimate the ratio of $\mathrm{Fe}^{3+}$ to $\mathrm{Fe}^{2+}$ in a mixed-species [5,6]. A smaller intensity indicates a 6-coordinate, e.g. octahedral $\left(O_{h}\right)$ system and a greater intensity corresponds to a 4-coordinate, e.g. tetrahedral $\left(T_{d}\right)$ compound. The high intensity of the pre-edge in a $T_{d}$ co-ordination occurs due to a transition from the $1 \mathrm{~s}$ orbital to the $\mathrm{p}$ component in a hybridised d-p orbital [44]. Hybridisation of the $3 d$ and $4 p$ orbitals does not occur in an $O_{h}$ compound and, instead, the pre-edge feature seen in the $\mathrm{Fe} K$-edge for $O_{h}$ co-ordination corresponds to the $1 \mathrm{~s} \rightarrow 3 \mathrm{~d}$ transition [45]. As a result, investigations of the pre-edge are useful for investigating the electronic transition that occur in iron minerals. Furthermore, it has been shown [46] that analysis of the pre-edge feature can be used to study archaeological iron corrosion and, in particular, to differentiate akaganeite and its precursor $\mathrm{Fe}(\mathrm{II})$ compound, $\beta-\mathrm{Fe}_{2}(\mathrm{OH})_{3} \mathrm{Cl}$, in a sample.

First, to establish the validity of the methodology, spectra from the $\mathrm{Fe}$ standards library were analysed using the developed fitting methodology, Figure $\mathbf{5}$ and Table 3 . Previously published pre-edge fits of iron minerals [5,6], have observed an energy gap of $1.4 \mathrm{eV}$ between $\mathrm{Fe}$ (II) and $\mathrm{Fe}$ (III) containing species; with centroid positions at 7112 and $7113.4 \mathrm{eV}$ respectively. In this work a similar gap is observed $(1.5 \mathrm{eV})$ between the two iron oxidation states, however they are found to occur at 7113 and $7114.5 \mathrm{eV}$ (see olive dashed line in Figure 5). This $\sim 1 \mathrm{eV}$ difference compared to the literature reference is due to the value of $\mathrm{E}_{0}$ used to calibrate the XANES spectra. Here, $\mathrm{E}_{0}$ for the $\mathrm{Fe} K$-edge is taken as $7112 \mathrm{eV}$, while Wilke et al. used a value of $7111.08 \mathrm{eV}$. Both are considered valid reference points for spectral calibration [47], however, this variation in calibration has resulted in a $\sim 1 \mathrm{eV}$ offset in absolute energy found in these data when compared to previous investigations.

In the library of fitted standards, the result of $\mathrm{FeCO}_{3}$ is anomalous. Though the phase is an Fe(II) species, it has a preedge appearance like $\mathrm{Fe}(\mathrm{HI})$, with an average centroid position of $7114.51 \mathrm{eV}$. When considering the rest of the absorption spectrum of the $\mathrm{FeCO}_{3}$ standard, the position of the white line (supplementary information S.2) is consistent with an $\mathrm{Fe}(\mathrm{II})$ oxidation state. This standard was synthesised in the laboratory via a precipitation mechanism from an $\mathrm{Fe}(\mathrm{II})$ solution. The pre-edge fit shows the peak corresponding to the $1 \mathrm{~s} \rightarrow 3 \mathrm{~d}$ transition [45] (peak 2) to be particularly strong. A possible explanation for the anomalous pre-edge character is a small amount of oxidation to Fe(III) during synthesis of the sample. Compared to other Fe(II) compounds, siderite has been observed to show the largest deviation from the average $\mathrm{Fe}(\mathrm{II})$ centroid position [5], which suggests a greater natural variation can occur for this phase than a typical Fe(II) species.

Comparing the results of the fitted pre-edges from the archaeological powders to the standards library, Figure 6-a, the majority of SS shot (purple stars) plot in the region between the iron oxyhydroxides and the two spinel phases. This is consistent with the SXPD and LCA phase analysis, which indicate the predominate composition is a mixture of goethite and magnetite/maghemite. Further supporting this, the SS powders that plot with the highest intensity correspond to the samples with the greatest spinel character (81A2618$\mathrm{C} 1$, intensity $=0.26$; and $82 \mathrm{~A} 2618-\mathrm{C} 4$, intensity $=0.22$ ), as derived from Figure 3. This can also be seen for the same phases in their $\chi(k)$ plot, Figure 2, bottom left, where a peak is present at $5.2 \AA^{-1}$ for the samples with a high concentration of magnetite.

In contrast to the SS shot, powder samples from $\mathrm{HW}$ and HWAS shot appear with a higher intensity and lower energy value than expected. As shown by the compositions in Figure 3, all HW and HWAS samples, except one (81A2798-P2), contain a metallic phase of either ferrite or cementite, whereas only 2 SS samples include a metal phase in the diffraction composition. A direct comparison between the metallic content of the samples and the pre-edge intensity, Figure 6-b, shows a positive correlation between pre-edge intensity and metallic character, particularly for the HW (yellow triangles) and HWAS (brown squares) samples. An examination of the metal standard spectra, Figure 7 (top), illustrates why the presence of $\mathrm{Fe}^{0}$ changes the pre-edge region of the samples. Both the $\alpha-\mathrm{Fe}$ foil and $\mathrm{Fe}_{3} \mathrm{C}$ standard show an intense "hump" between 7105 and $7118 \mathrm{eV}$. Unfortunately, this overlaps with the region associated with the iron pre-edge feature, which appears between 7110 and $7120 \mathrm{eV}$. The additional contribution from a metallic phase, therefore, results in an increase in intensity and reduction in energy of any pre-edge feature extracted from the spectrum. Consequently, it is concluded that the pre-edge analysis methodology is inappropriate for a mixed metal-oxide system. Therefore, if future work looked to apply a similar pre-edge analysis to mixed-phase Fe XANES spectra from corrosion systems, sampling approaches should be adapted to prevent inclusion of the metal substrate in the sample.

\subsection{TEY spectra from cross-section samples}

Alongside the surface and cutting powders, 2 cross-section (-S) samples were prepared for each treatment type, giving a total of 6 -S samples for analysis. To investigate how the 
chemistry of the iron species changes spatially across the artefacts, a series of XAS spectra were collected from the cross-section samples at the Fe $K$-edge, Figure 7, in line scans from the outer to inner region of the cross-section. Looking at the XANES spectral series from the external surface of the artefact (green spectra), to the internal metallic regions (purple spectra), a transition occurs from more oxidic features to more metallic features. Specifically, the purple spectra have a broader, more intense "hump" at $\sim 710 \mathrm{eV}$, while the green spectra show a more pre-edge like feature in this region. Further, at the peak of the white line at $\sim 7130 \mathrm{eV}$, the green spectra are more intense and narrower in shape. Comparing these regions to the metal and oxide standards most commonly identified in the shot during analysis of the powder samples $\alpha-\mathrm{Fe}, \mathrm{Fe}_{3} \mathrm{C}, \mathrm{Fe}_{3} \mathrm{O}_{4}$ and $\alpha-\mathrm{FeO}(\mathrm{OH})$ - these changes are consistent with increased oxide character towards the outer surface, a trend which is expected for a corroded metal alloy. This transition is best observed for the SS treated shot 82A2618 (Figure 7, top right), where a clear distinction can be seen between the metallic areas at 50,50 and 60,60 px and the oxide regions from $70,70 \mathrm{px}$ onwards. This sample presented with the thickest $(\sim 8 \mathrm{~mm})$ oxide layer of all the objects studied and was found to be particularly well-suited to cross-section XAS analysis. A detailed examination of the XANES spectra from cross section $82 \mathrm{~A} 2618$-S8 by LCA supplementary information S.5 - shows that the corrosion system consists predominantly of magnetite and goethite in varying compositions across the artefact. These form distinct layers within the cross-section. Beginning at the metal/corrosion interface, the layers comprised $1 \mathrm{~mm} \mathrm{Fe}_{3} \mathrm{O}_{4}$, $3 \mathrm{~mm} \alpha-\mathrm{FeO}(\mathrm{OH}), 3 \mathrm{~mm} \mathrm{Fe}_{3} \mathrm{O}_{4}$, and finally $1 \mathrm{~mm}$ of a complex outer surface, which could not be reliably identified by the LCA analysis. In contrast, the other 5 samples have much thinner corrosion layers, generally $<0.5 \mathrm{~mm}$ and have greater metallic character at every point in the depth-profiling spectral series. As discussed in the previous sections, and as seen in the table in supplementary information S.5, a greater metallic character to the XAS spectrum results in a lower quality fit using LCA. Consequently, the presence of metallic iron in the XAS data from the remaining 5 -S samples means that their analysis has been limited to spectral fingerprinting.

\section{Conclusions}

This work used a combination of linear combination analysis, LCA, and pre-edge fitting to analyse the $\mathrm{Fe} K$-edge XANES spectra of 30 iron corrosion powders (-C and -P samples) and 6 cross-sections (-S samples) with reference to a library of standard iron corrosion products. Comparison of the phase quantification of the best fit LCA results to the compositions derived from diffraction data show that XAS and SXPD are highly complementary for investigating samples comprised of a mixture of iron oxides and/or oxyhydroxides. However, each technique presents limitations in phase identification, either between magnetite and maghemite for SXPD or in differentiating metallic phases with XAS LCA, emphasising the benefit of using the methods together. Both methods allow the identification of the phase akaganeite which, due to its incorporation of chloride, is strongly associated with the corrosion of archaeological iron $[4,33,35,38]$. The XANES data confirm previous observations that akaganeite is absent in the corrosion layers of passively stored archaeological iron but is generated after removal and during active conservation [19]. As chloride may accelerate or trigger further corrosion reactions [48], conservation strategies are required which minimise akaganeite formation.

The present results indicate that, if any metallic iron - either in the form of $\alpha$-Fe or $\mathrm{Fe}_{3} \mathrm{C}-$ is present in the sample, characterisation of the XANES spectra becomes more challenging and the results are less reliable. This conclusion was reflected in the pre-edge analysis, where the methodology was shown to be effective for predicting the co-ordination and oxidation of the mixed-phase corrosion samples that did not have any metal character, but the approach was found to be inappropriate for any sample with metallic iron phases in the mixture. Specifically, in the presence of metallic iron, the intensity and energy of the pre-edge feature cannot be robustly identified, meaning it is not possible to derive the ratio of $\mathrm{Fe}^{2+}$ to $\mathrm{Fe}^{3+}$

The corrosion of archaeological iron remains one of the most challenging problems in heritage conservation and further synchrotron-based investigations in this area are anticipated. From the present investigation, it has been demonstrated that while XAS data analysis methodologies developed for single phase-systems can be applied to complex, naturally formed samples, they cannot be used universally. Future investigations incorporating $\mathrm{Fe} K$-edge XAS analysis into an iron corrosion study should take steps to minimise the metallic component of the samples to maximise the conclusions that may be drawn from the data. Furthermore, the use of a complementary phase identification technique such as XRD, is strongly recommended. Finally, it is noted that these findings are not only pertinent to heritage science but may also be of use in corrosion research or in environmental studies involving multi-phase iron oxide samples.

\section{Acknowledgements}

The work presented in this paper is co-funded by the UK's Engineering and Physical Sciences Research Council (EPSRC) and Diamond Light Source as part of the Centre for Doctoral Training in Science and Engineering in Arts, Heritage and Archaeology (SEAHA-CDT). Data included in this manuscript was collected at Diamond Light Source, as part of the beamtime session SP16328. The authors wish to thank Mr Phil Robbins, Dr Sarah Day, Dr Chiu Tang, Dr 
Steven Parry, Dr Diego Gianolio and Ms Ann-Kathrin Geiger for their helpful contributions to this project.

\section{References}

[1] Cornell R and Schwertmann U 2003 The Iron Oxides: Structure, Properties, Reactions, Occurrences and Uses, (Weinheim, Germany: Wiley-VCH Verlag GmbH \& Co. $\mathrm{KGaA}$ )

[2] Neff D, Bellot-Gurlet L, Dillmann P, Reguer S and Legrand L 2006 Raman imaging of ancient rust scales on archaeological iron artefacts for long-term atmospheric corrosion mechanisms study J. Raman Spectrosc. 37 122837

[3] Neff D, Dillmann P, Bellot-Gurlet L and Beranger G 2005 Corrosion of iron archaeological artefacts in soil: Characterisation of the corrosion system Corros. Sci. 47 515-35

[4] Rémazeilles C, Neff D, Kergourlay F, Foy E, Conforto E, Guilminot E, Reguer S, Refait P and Dillmann P 2009 Mechanisms of long-term anaerobic corrosion of iron archaeological artefacts in seawater Corros. Sci. 512932 41

[5] Wilke M, Farges F, Petit P-E, Brown G E and Martin F 2001 Oxidation state and coordination of $\mathrm{Fe}$ in minerals: An Fe K- XANES spectroscopic study Am. Mineral. 86 714-30

[6] Petit P, Farges F, Wilke M and Sole V A 2001 Determination of the iron oxidation state in Earth materials using XANES pre-edge information environmental, earth and planetary science J. Synchrotron Radiat. 8 952-4

[7] Wilke M, Farges F, Partzsch G M, Schmidt C and Behrens $\mathrm{H} 2007$ Speciation of $\mathrm{Fe}$ in silicate glasses and melts by insitu XANES spectroscopy Am. Mineral. 92 44-56

[8] Wilke M, Partzsch G M, Bernhardt R and Lattard D 2005 Determination of the iron oxidation state in basaltic glasses using XANES at the K-edge Chem. Geol. 220 143-61

[9] Jaklevic J M and Thompson A C 1982 X-ray methods for the chemical characterization of atmospheric aerosols" Nucl. Instruments Methods 193 309-14

[10] Waychunas G A, Apted M J and Brown GE 1983 X-ray K-edge absorption spectra of Fe minerals and model compounds: Near-edge structure Phys. Chem. Miner. $101-$ 9

[11] SAKURAI K, IIDA A and GOHSHI Y 1988 Chemical State Analysis by X-Ray Fluorescence Using Shifts of Iron K Absorption Edge Anal. Sci. 4 37-41

[12] GURMAN S J, GLADDEN L F, SPENCE C A and COX P 1988 A STRUCTURAL BASIS FOR THE CORROSION-RESISTANCE OF LEAD-IRONPHOSPHATE GLASSES - AN X-RAY ABSORPTIONSPECTROSCOPY STUDY Philos. Mag. B-PHYSICS Condens. MATTER Stat. Mech. Electron. Opt. Magn. Prop. 58 271-83

[13] Kerkar M, Robinson J and Forty A J 1990 In situ structural studies of the passive film on iron and iron/chromium alloys using X-ray absorption spectroscopy Faraday Discuss. Chem. Soc. 89 31-40

[14] Nakai I, Taguchi I and Yamasaki K 1991 Chemical speciation of archaeological objects by XRF/XANES analysis using synchrotron radiation Anal. Sci. 7 365-8

[15] Kendig M W, Davenport A J and Isaacs H S 1993 The mechanism of corrosion inhibition by chromate conversion coatings from $\mathrm{x}$-ray absorption near edge spectroscopy
[16] Ganes) Corros. Sci. 34 41-9 Marcelli A 2020 Accurate Fe3+/Fetot ratio from XAS spectra at the Fe K-edge Radiat. Phys. Chem. 175108088

[17] Dyar M D, Breves E A, Gunter M E, Lanzirotti A, Tucker J M, Carey C J, Peel S E, Brown E B, Oberti R, Lerotic M and Delaney J S 2016 Use of multivariate analysis for synchrotron micro-XANES analysis of iron valence state in amphiboles Am. Mineral. 101 1171-89

[18] Jackson W E, Farges F, Yeager M, Mabrouk P A, Rossano S, Waychunas G A, Solomon E I and Brown G E 2005 Multi-spectroscopic study of $\mathrm{Fe}$ (II) in silicate glasses: Implications for the coordination environment of $\mathrm{Fe}$ (II) in silicate melts Geochmica Cosmochim. Acta 69 4315-32

[19] Simon H, Cibin G, Robbins P, Day S, Tang C, Freestone I and Schofield E 2018 A Synchrotron-Based Study of the Mary Rose Iron Cannonballs Angew. Chemie - Int. Ed. 57 7390-5

[20] Simon H J, Cibin G, Reinhard C, Liu Y, Schofield E and Freestone I C 2019 Influence of microstructure on the corrosion of archaeological iron observed using 3D synchrotron micro-tomography Corros. Sci. 159108132

[21] Hildred A 2011 Weapons of Warre: The Armaments of the Mary Rose (Exeter: The Mary Rose Trust Ltd)

[22] Jones M 2003 For Future Generations: Conservation of a Tudor Maritime Collection ed M Jones (Portsmouth: The Mary Rose Trust Ltd)

[23] Marsden P 2003 Sealed by Time: the loss and recovery of the Mary Rose (Portsmouth: The Mary Rose Trust Ltd)

[24] Argyropoulos V, Rameau J-J, Dalard F and Degrigny C 1999 Testing Hostacor IT as a corrosion inhibitor for iron in polyethylene glycol solutions Stud. Conserv. 44 49-57 North N A and Pearson C 1975 Alkaline sulfite reduction treatment of marine iron Icom committee for conservation. 4th triennial meeting, Venice, 13-18 October. Preprints (International Council of Museums) pp 75131-3 Dent A J, Cibin G, Ramos S, Smith A D, Scott S M, Varandas L, Pearson M R, Jones C P and Robbins P E 2009 B18: A core XAS spectroscopy beamline for Diamond 14th International Conference on X-Ray Absorption Fine Structure (XAFS14) pp 1-4

[27] Dent A J, Cibin G, Ramos S, Parry S A, Gianolio D, Smith A D, Scott S M, Varandas L, Patel S, Pearson M R, Hudson L, Krumpa N A, Marsch A S and Robbins P E 2013 Performance of B18, the Core EXAFS Bending Magnet beamline at Diamond 15th International Conference on X-Ray Absorption Fine Structure (XAFS15) pp 1-7

[28] Anné J, Edwards N P, van Veelen A, Egerton V M, Manning P L, Mosselmans J F W, Parry S, Sellers W I, Buckley M and Wogelius R A 2017 Visualisation of developmental ossification using trace element mapping $J$. Anal. At. Spectrom.

[29] Ravel B and Newville M 2005 ATHENA, ARTEMIS, HEPHAESTUS : data analysis for X-ray absorption spectroscopy using IFEFFIT J. Synchrotron Radiat. 12 537-41

[30] Remazeilles C, Saheb M, Neff D, Guilminot E, Tran K, Bourdoiseau J A, Sabot R, Jeannin M, Matthiesen H, Dillmann P and Refait P 2010 Microbiologically influenced corrosion of archaeological artefacts: characterisation of iron (II) sulfides by Raman spectroscopy J. Raman Spectrosc. 41 1425-33

[31] North N A 1982 Corrosion products on marine iron Stud. 
Conserv. 27 75-83

[32] North N A 1976 Formation of coral concretions on marine iron Int. J. Naut. Archaeol. Underw. Explor. $5.3253-8$

[33] Selwyn L 2004 Overview of archaeological iron: the corrosion problem, key factors affecting treatment, and gaps in current knowledge Proc. Met. 2004 294-306

[34] Turgoose S 1985 The corrosion of archaeological iron during burial and treatment Stud. Conserv. 30 13-8

[35] Reguer S, Kergourlay F, Foy E, Neff D, Vantelon D, Cotte M, Mirambet F and Dillmann P 2020 XANES at the Cl Kedge as a relevant technique to reveal the iron archaeological artefact dechlorination treatments J. Anal. At. Spectrom. 35 2358-68

[36] Kergourlay F, Réguer S, Neff D, Foy E, Picca F, Saheb M, Hustache S, Mirambet F and Dillmann P 2018

Stabilization treatment of cultural heritage artefacts: In situ monitoring of marine iron objects dechlorinated in alkali solution Corros. Sci. 132 21-34

[37] Réguer S, Mirambet F, Rémazeilles C, Vantelon D, Kergourlay F, Neff D and Dillmann P 2015 Iron corrosion in archaeological context: Structural refinement of the ferrous hydroxychloride b-Fe2(OH)3Cl Corros. Sci. 100 589-98

[38] Ståhl K, Nielsen K, Jiang J, Lebech B, Hanson J C, Norby $\mathrm{P}$ and van Lanschot $\mathbf{J} 2003$ On the akaganéite crystal structure, phase transformations and possible role in postexcavational corrosion of iron artifacts Corros. Sci. $\mathbf{4 5}$ 2563-75

[39] Cibin G, Gianolio D, Parry S A, Schoonjans T, Moore O, Draper R, Miller L A, Thoma A, Doswell C L and Graham A 2020 An open access, integrated XAS data repository at Diamond Light Source Radiat. Phys. Chem. 175108479

[40] Michelin A, Drouet E, Foy E, Dynes J J, Neff D and Dillmann P 2013 Investigation at the nanometre scale on the corrosion mechanisms of archaeological ferrous artefacts by STXM J. Anal. At. Spectrom. 28 59-66

[41] Leon Y, Saheb M, Drouet E, Neff D, Foy E, Leroy E, Dynes J J and Dillmann P 2014 Interfacial layer on archaeological mild steel corroded in carbonated anoxic environments studied with coupled micro and nano probes Corros. Sci. 88 23-35

[42] Neff D, Dillmann P and Reguer S 2013 Analytical techniques for the study of corrosion of metallic heritage artifacts: from micrometer to nanometer scales Corrosion and Conservation of Cultural Heritage Metallic Artefacts ed P Dillmann, D Watkinson, E Angelini and A Adriaens (Woodhead Publishing Limited) pp 55-81

[43] Farges F, Lefrere Y, Rossano S, Berthereau A, Calas G and Brown G E 2004 The effect of redox state on the local structural environment of iron in silicate glasses: a combined XAFS spectroscopy, molecular dynamics, and bond valence study J. Non. Cryst. Solids 344 176-88

[44] Yamamoto T 2008 Assignment of pre-edge peaks in Kedge $\mathrm{x}$-ray absorption spectra of $3 \mathrm{~d}$ transition metal compounds: electric dipole or quadrupole? $X$-ray Spectrom. 37 572-84

[45] Galoisy L, Calas G and Arrio M A2001 High-resolution XANES spectra of iron in minerals and glasses structural information from the pre-edge region Chem. Geol. 174 307-19

[46] Réguer S, Dillmann P and Mirambet F 2007 Buried iron archaeological artefacts: Corrosion mechanisms related to the presence of Cl-containing phases Corros. Sci. $492726-$ 44

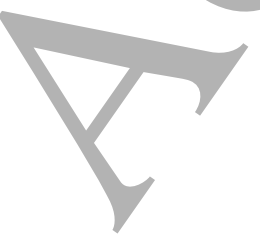

[47] Calvin S 2013 XAFS for everyone (Boca Raton, FL: CRC Press)

[48] Rimmer M, Watkinson D and Wang Q 2013 The impact of chloride desalination on the corrosion rate of archaeological iron Stud. Conserv. 58 326-37

[49] Vidyasagar K, Gopalakrishnan J and Rao C 1984 A convenient route for the synthesis of complex metal oxides employing solid-solution precursors Inorg. Chem. 23 1206-10

[50] Gotor F J, Macías M, Ortega A and Criado J M 2000 Comparative study of the kinetics of the thermal decomposition of synthetic and natural siderite samples Phys. Chem. Miner. 27 495-503

[51] Cornell R M and Schwertmann U 2000 Maghemite Iron Oxides in the Laboratory: Preparation and Characterization (Weinheim, Germany: Wiley VCH Verlag GmbH) pp 141-2

[52] Refait P and Genin J M. 1997 The Mechanisms of Oxidation of Ferrous Hydroxychloride b- $\mathrm{Fe} 2(\mathrm{OH}) 3 \mathrm{Cl}$ in Aqueous Solution: The Formation of Akaganeite vs Goethite Corros. Sci. 39 539-53 
Table 1 Library of Fe corrosion standards

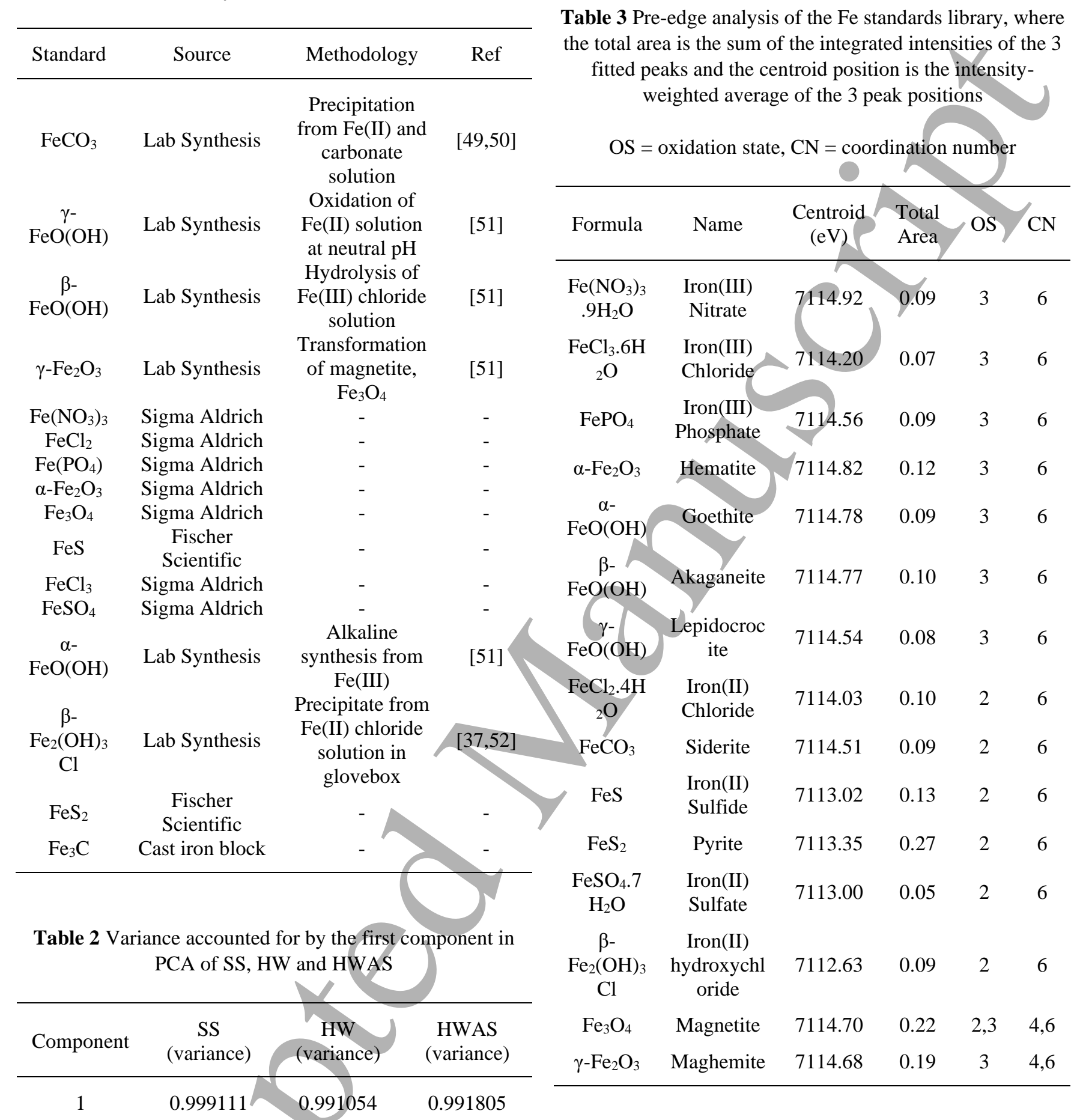


Figure 1 Cubic spline model used to extract pre-edge feature from Fe k-edge XANES data (left) and resulting backgroundsubtracted pre-edge (right)
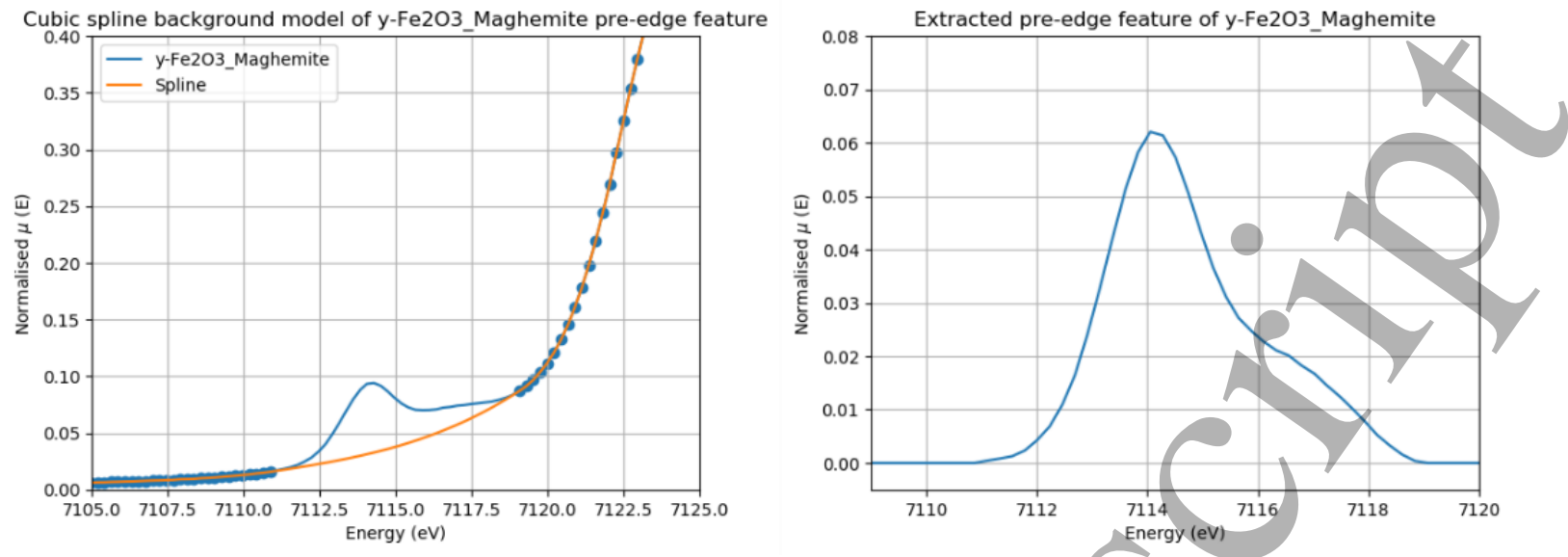

Figure 2 Fe $K$-edge XAS of $-\mathrm{C}$ and -P samples from SS, HW and HWAS. Top normalised $\mu(\mathrm{E})$ in energy space bottom EXAFS, $\chi(\mathrm{k})$, in k-space with a k-weighting of 2. One additional powder sample (81A3839-C2) was measured by XAS but not SXPD, the sample spectrum is shown here, but is not included in the analysis in Figures 3, 4 and 7. Axis labels are the same for all 3 treatment types, but only shown for SS for brevity.

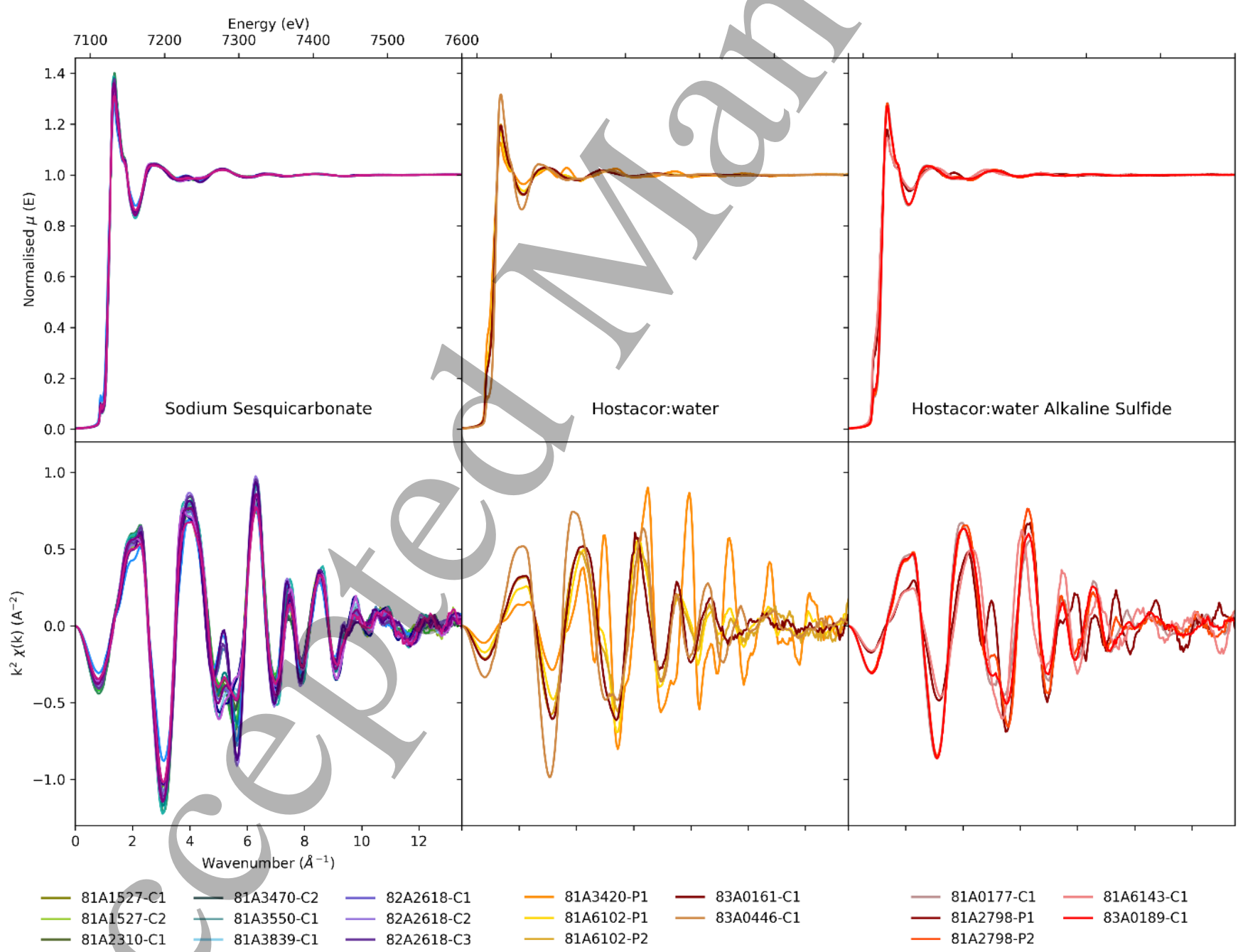


Figure 3 Phase proportion of iron species calculated by a: XAS LCA and b: SXPD.
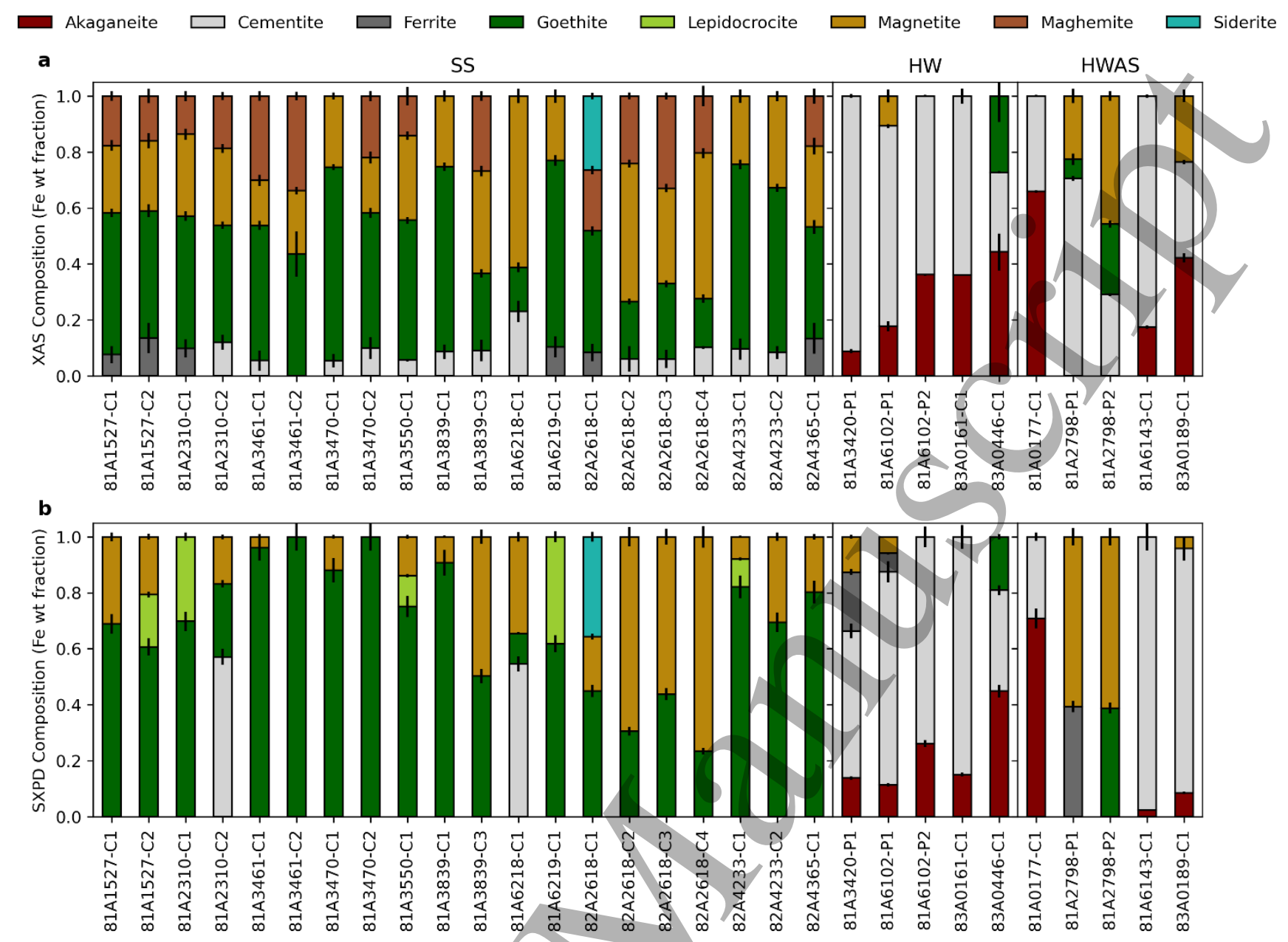

Figure 4 Comparison of XAS and SXPD iron phase compositions for SS, HW and HWAS. For comparison purposes, the XAS results for magnetite and maghemite have been combined and are referred to as 'magnetite' as the wt fraction of the 'magnetite' structure in SXPD represents both species. Pink dashed line corresponds to an equal XAS and SXPD phase composition

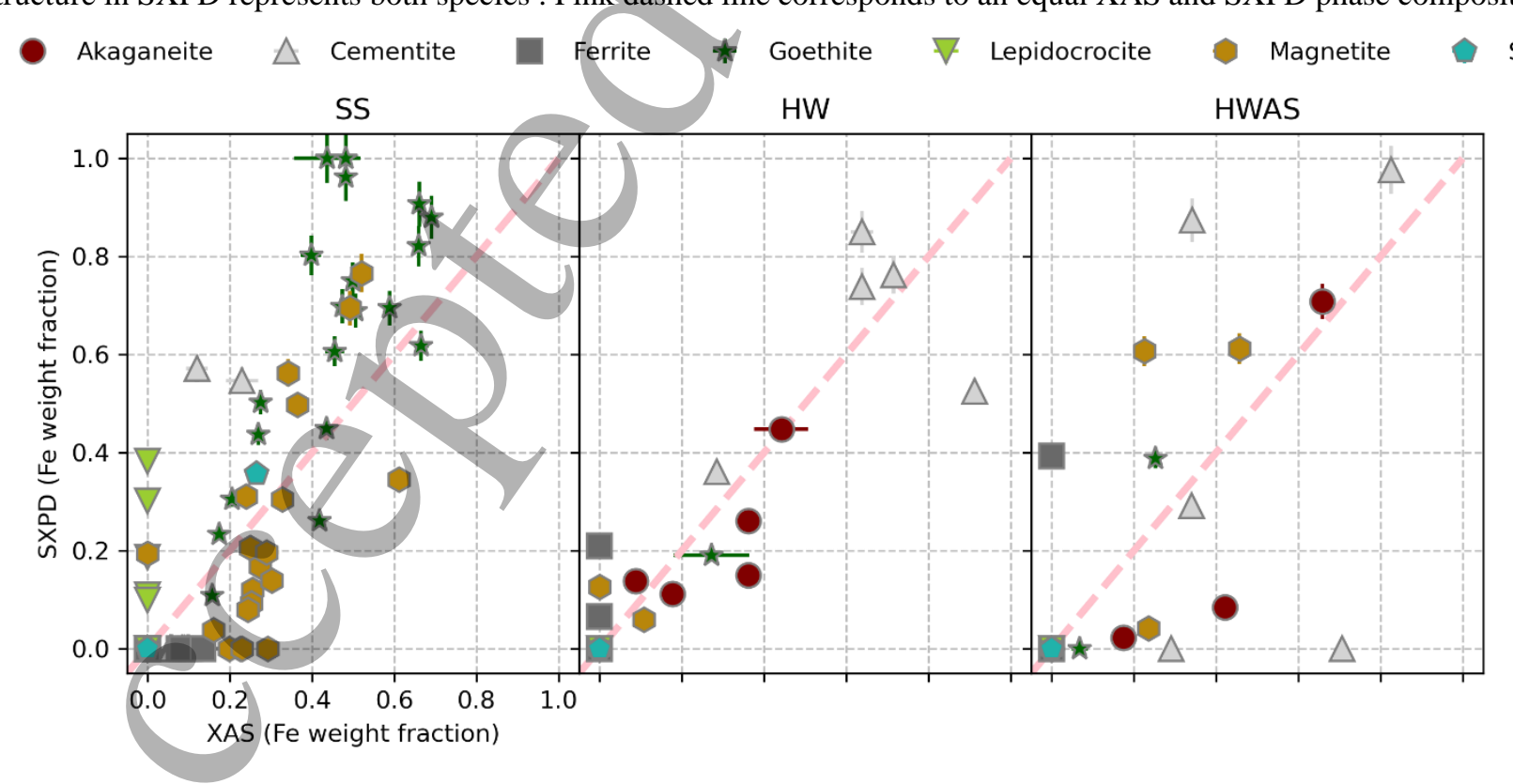


Figure 5 Extracted pre-edges of iron standards library (blue line) and 3-peak fit (dashed grey) for a: Fe(III), b: Fe(II) and c: spinel phases. Dashed olive line shows the average position of the $\mathrm{Fe}$ (II) and Fe(III) centroids at 7113 and $7114.5 \mathrm{eV}$ respectively
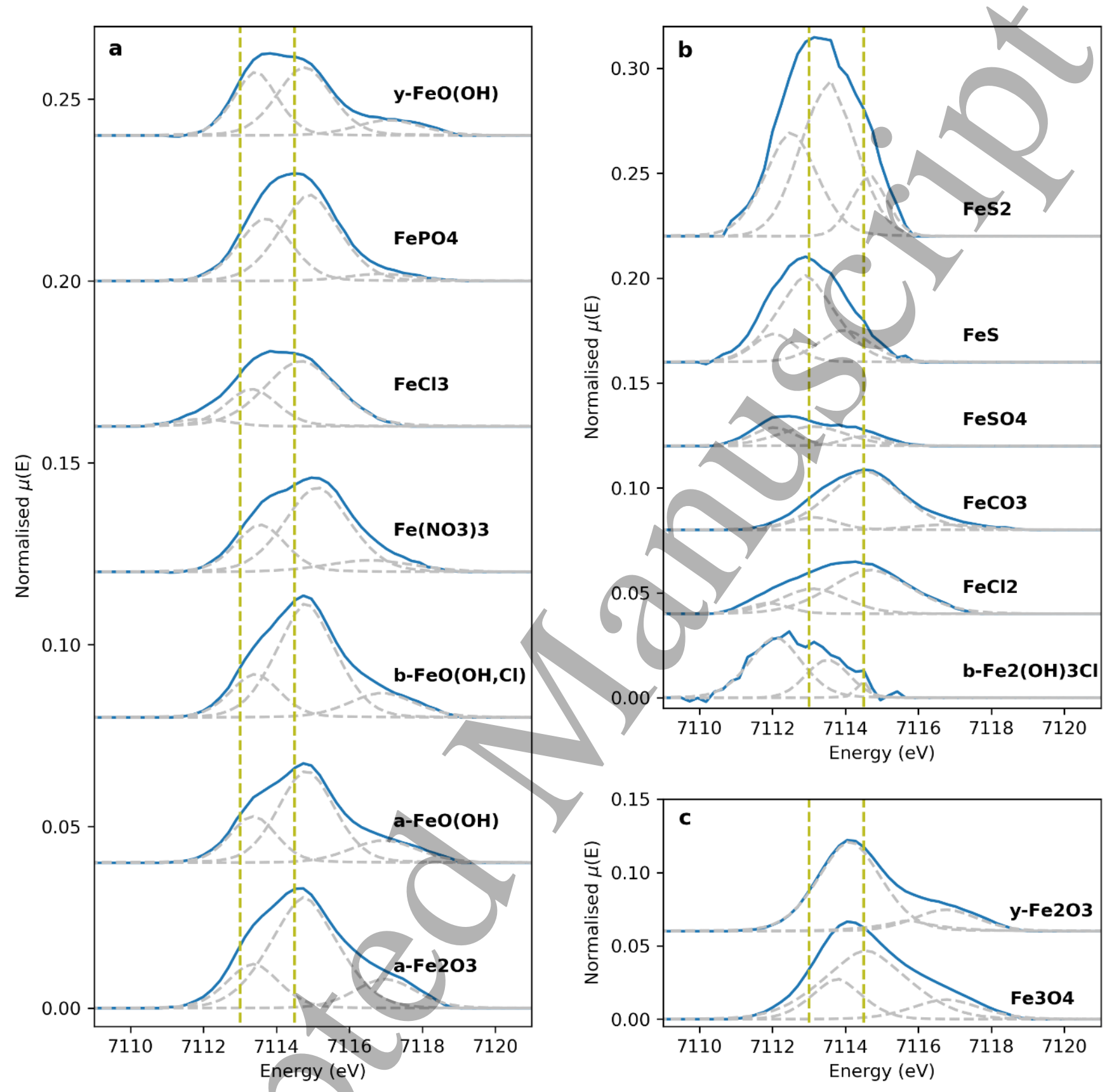
Figure 6 a: Plot of pre-edge intensity vs. average centroid position for standards shown as circles (Fe(III) - red, Fe(II) - green, spinel - blue) and samples (SS - purple stars, HW - yellow triangles, HWAS - brown squares). Dashed olive vertical lines show the average position of the $\mathrm{Fe}(\mathrm{II})$ and $\mathrm{Fe}(\mathrm{III})$ centroids at 7113 and $7114.5 \mathrm{eV}$ respectively; b: Comparison of metal ( $\mathrm{Fe}_{3} \mathrm{C}$ and $\alpha-\mathrm{Fe}$ ) content of archeological samples determined from diffraction to the integrated pre-edge intensity. Dashed green line shows a linear regression fit to the data.
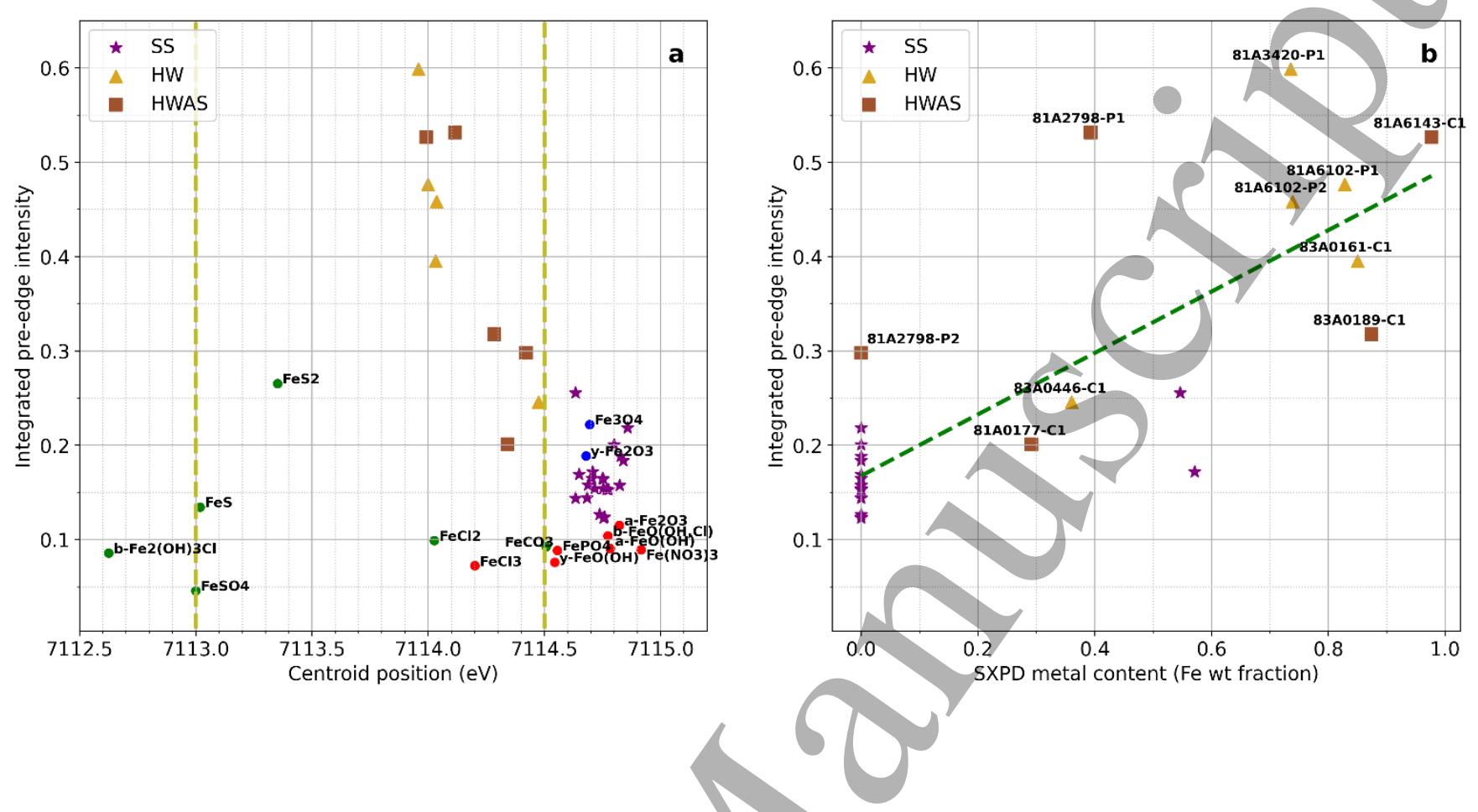
Figure 7 Fe $K$-edge of cross-section (-S) samples going from the outer, external surface (green) to the inner, metallic region of the artefact (purple) for 6 shot treated by: Sodium Sesquicarbonate (first row), Hostacor:Water (second row) and Hostacor:Water Alkaline Sulfide (third row). A plot of relevant standards is given above the archaeological datasets to assist assignment of spectral features. Each Mary Rose sample is plotted with an inset showing the Fe XRF elemental map for the sample, with the locations of data acquisition indicated as white stars.

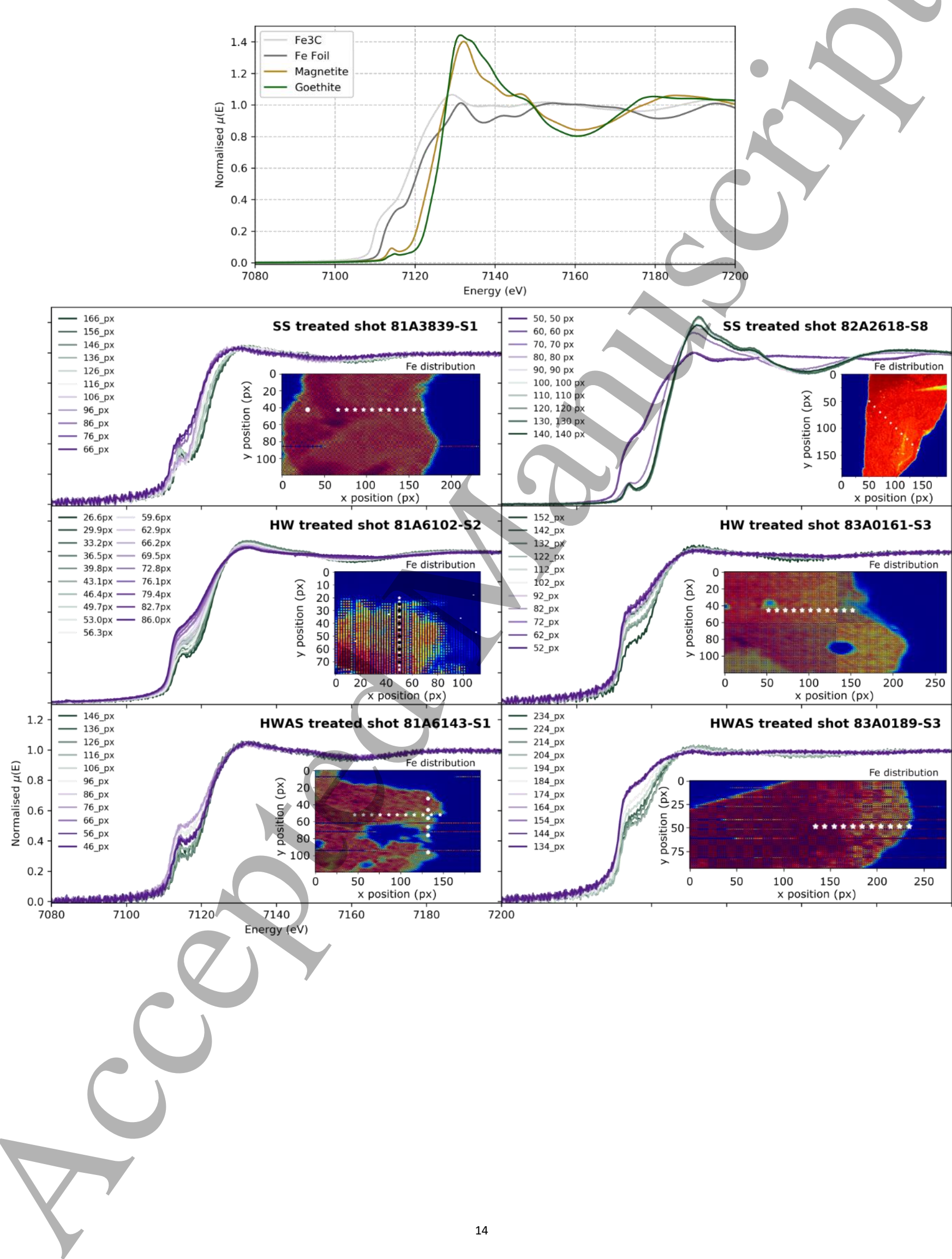

